FERNANDES, A.A.; MARTINEZ, H.E.P.; FONTES, P.C.R. Produtividade, qualidade dos frutos e estado nutricional do tomateiro tipo longa vida conduzido com um cacho, em cultivo hidropônico, em função das fontes de nutrientes. Horticultura Brasileira, Brasília, v. 20, n. 4, p. 564-570, dezembro 2002.

\title{
Produtividade, qualidade dos frutos e estado nutricional do tomateiro tipo longa vida conduzido com um cacho, em cultivo hidropônico, em função das fontes de nutrientes
}

\author{
Adriano A. Fernandes; Herminia Emilia P. Martinez; Paulo Cesar R. Fontes \\ UFV, Depto. Fitotecnia, 36.571-000 Viçosa-MG; E-mail: aalves@alunos.ufv.br
}

\begin{abstract}
RESUMO
Avaliou-se a produtividade, qualidade dos frutos e estado nutricional do tomateiro longa vida, conduzido com um cacho e cultivado em soluções nutritivas preparadas com diferentes conjuntos de fontes de nutrientes. $O$ experimento foi conduzido de 04/05/99 a 13/09/99 em casa de vegetação da UFV. Foram avaliados dois conjuntos de fontes de nutrientes para compor as soluções de crescimento vegetativo e dois para as soluções de frutificação. As combinações desses conjuntos deram origem a quatro tratamentos que foram dispostos no delineamento de blocos ao acaso com oito repetições. As mudas de tomate, híbrido Carmen, com 36 dias de idade foram colocadas em vasos de 8,6 L, com espaçamento de $0,35 \mathrm{x}$ $0,70 \mathrm{~m}$. Foi feita análise foliar para determinar o estado nutricional da planta. Avaliou-se também a partição dos nutrientes $\mathrm{Ca}, \mathrm{Mg}$ e K no fruto. Os dados obtidos foram submetidos à análise de variância e as médias dos tratamentos, comparadas pelo teste de Tukey a 5\% de probabilidade. Para as características avaliadas não foram encontradas diferenças significativas entre os tratamentos. A produção média de frutos por planta foi de $920 \mathrm{~g}$, com ciclo de 96 dias, o que corresponderia no espaçamento adotado à produtividade média de $142 \mathrm{t} \mathrm{ha}^{-1}$ ano $^{-1}$. A concentração de $\mathrm{N}, \mathrm{P}, \mathrm{K}, \mathrm{Ca}, \mathrm{Mg}$ e S, nas folhas foram, respectivamente, 3,$18 ; 1,25 ; 5,11 ; 4,50 ; 0,88$; e 1,79 dag kg1, e as concentrações de $\mathrm{Fe}, \mathrm{Zn}, \mathrm{Cu}, \mathrm{Mn}$ e $\mathrm{B}$ foram, respectivamente, 209; 96; 10; 665; e $209 \mathrm{mg} \mathrm{kg}^{-1}$. No fruto o Ca e K apresentaram maior concentração na região proximal ao pedúnculo, ao contrário do $\mathrm{Mg}$, que nessa porção apresentou menor concentração.
\end{abstract}

Palavras-chave: Lycopersicon esculentum L., soluções nutritivas, nutrição mineral.

\begin{abstract}
Productivity, fruit quality and nutritional status of single truss long shelf life tomato, cultivated in hydroponic system, with different nutrient sources

There were evaluated the yield, fruit quality and nutritional status of single truss and long shelf life tomato, cultivated in nutritive solutions prepared with different nutrient sources. The experiment was carried out from $04 / 05 / 99$ to $13 / 09 / 99$ in a greenhouse of the Universidade Federal de Viçosa, Brazil. Two groups of nutrient sources were tested to prepare the solutions for vegetative growth and two groups for the frutification solutions. The combinations of those groups gave rise to four treatments, disposed in the randomized blocks design with eight repetitions. Seedlings of the $F_{1}$ hybrid Carmen 36-day old were placed in 8,6 L vases, spaced $0.35 \times 0.70$ $\mathrm{m}$. The yield, fruit quality and nutritional status of the plants were evaluated, as well as the partition of $\mathrm{Ca}, \mathrm{Mg}$ and $\mathrm{K}$ in the fruit. The data were submitted to analysis of variance and the means were discriminated using Tukey at $5 \%$ of probability. There were no significant differences among treatments. The average yield per plant was of $920 \mathrm{~g}$, with an average plant cycle of 96 days, corresponding to a yield of $142 \mathrm{t} \mathrm{ha}^{-1}$ year $^{-1}$. The concentrations of $\mathrm{N}, \mathrm{P}, \mathrm{K}, \mathrm{Ca}, \mathrm{Mg}$ and $\mathrm{S}$ in leaves were, respectively, $3.18 ; 1.25 ; 5.11 ; 4.50 ; 0.88$; and $1.79 \mathrm{dag} \mathrm{kg}^{-1}$, and concentrations of $\mathrm{Fe}, \mathrm{Zn}, \mathrm{Cu}, \mathrm{Mn}$ and $\mathrm{B}$ were, respectively, 209; 96; 10;665; and $209 \mathrm{mg} \mathrm{kg}^{-1}$. In the fruit, $\mathrm{Ca}$ and $\mathrm{K}$ were presented in larger concentration in the proximal area, unlike $\mathrm{Mg}$, that was present only in lower concentration in that portion.
\end{abstract}

Keywords: Lycopersicon esculentum L., nutritive solutions, mineral nutrition.

(Recebido para publicação em 13 de dezembro de 2000 e aceito em 10 de outubro de 2002)

\begin{abstract}
A produção de hortaliças de frutos em cultivo hidropônico representa uma nova etapa. A princípio, somente hortaliças de folhas vinham sendo cultivadas nesse sistema (Moraes \& Furlani, 1999). Atualmente, devido à necessidade premente do mercado consumidor, os produtores buscam diversificar a produção. No Brasil, o cultivo hidropônico de frutos tem apresentado produtividade superior à atingida em cultivos tradicionais e em estufas-solo. (Moraes \& Furlani, 1999). Dentre as hortaliças de frutos mais cultivadas no Brasil, destacam-se principalmente os híbridos de tomateiro dos tipos caqui, cereja e longa vida. Pela sua grande acei-
\end{abstract}

tação no mercado e preços compensadores, o tomate tem sido, dentre as hortaliças de frutos, a de maior interesse por parte dos produtores.

No segmento do tomate in natura o setor continua a modernizar-se. Cresce bastante a produção de tomate do tipo longa-vida, havendo estimativas de que este já corresponderia a mais de $50 \%$ do volume comercializado nas grandes redes de supermercados. Para os produtores que atuam nesse mercado, o grande desafio continua sendo a comercialização. Os que conseguem fugir dos tradicionais canais de vendas e agregam valor ao produto normalmente obtêm excelentes lucros (Nakamae \& Pastrello, 1999).
Em países onde o cultivo hidropônico é bem desenvolvido, como Holanda e Japão, as altas produtividades alcançadas para a cultura do tomate nem sempre são acompanhadas por qualidade correspondente. Como a cultura tem ciclo longo, os riscos de aparecimento e disseminação de doenças são maiores. O cultivo do tomate com apenas um cacho por planta, reduz o ciclo da cultura, expondo-a a menores riscos de contaminação. Além disso, tal sistema é altamente produtivo e permite ajustes das concentrações de nutrientes e da condutividade elétrica da solução nutritiva de modo a obter um produto uni- 
forme e de alta qualidade, já que não se tem frutos em estádios fenológicos tão distintos (Morgan, 1996; Morgan, 1997a).

Diversos trabalhos têm mostrado que a qualidade de tomates cultivados em hidroponia depende diretamente da adequação da solução nutritiva empregada à fase de desenvolvimento da cultura (Adams, 1994). A partir do início da frutificação, a relação $\mathrm{N}: \mathrm{K}$ absorvidos altera-se drasticamente, sendo necessário o aumento do suprimento de $\mathrm{K}$ para garantir a qualidade e uniformidade de maturação dos frutos (Adams \& Massey, 1984; Ho \& Adams, 1995).

A composição ideal de uma solução nutritiva depende não somente das concentrações dos nutrientes, mas também de outros fatores ligados ao cultivo, incluindo o tipo de sistema hidropônico, os fatores ambientais, a época do ano, o estádio fenológico, a espécie vegetal e a cultivar em produção (Furlani et al., 1999a). Avaliou-se a produtividade, qualidade dos frutos e estado nutricional do tomateiro tipo longa vida cultivado em soluções nutritivas preparadas com diferentes conjuntos de fontes de nutrientes e conduzido com apenas um cacho.

\section{MATERIAL E MÉTODOS}

O experimento foi conduzido de 04/ 05 a 13/09/99 em estufa da UFV. Foram testados dois conjuntos de fontes de nutrientes na composição das soluções utilizadas na fase de crescimento vegetativo e dois conjuntos de fontes na composição das soluções utilizadas na fase de frutificação (Tabela 1). Ambas soluções utilizadas na fase de crescimento vegetativo continham: $8 ; 2 ; 4 ; 2$; 1; e $1 \mathrm{mmol} \mathrm{L}^{-1}$ de N, P, K, Ca, Mg e S e 35; 19; 21; 4; 0,9; e 0,7 mmol L-1 de Fe, $\mathrm{Mn}, \mathrm{B}, \mathrm{Zn}, \mathrm{Cu}$ e Mo, respectivamente. Ambas soluções utilizadas na fase de frutificação continham: $12 ; 3 ; 8,6 ; 3$; 1,5; e $1,5 \mathrm{mmol} \mathrm{L}^{-1}$ de N, P, K, Ca, Mg e $\mathrm{S}$ e $59 ; 28 ; 31 ; 4 ; 1,3$; e $0,7 \mathrm{mmol} \mathrm{L}^{-1}$ de $\mathrm{Fe}, \mathrm{Mn}, \mathrm{B}, \mathrm{Zn}, \mathrm{Cu}$ e Mo, respectivamente. As combinações desses conjuntos, expostos na Tabela 1 , deram origem a quatro tratamentos (Trat. $1=$ Conjunto $\mathrm{A}+\mathrm{C}$; Trat. $2=$ Conjunto $\mathrm{A}+\mathrm{D}$; Trat. 3 $=$ Conjunto $\mathrm{B}+\mathrm{C}$; Trat. $4=$ Conjunto $\mathrm{B}$ $+\mathrm{D}$;), que foram dispostos no delinea- mento de blocos ao acaso com oito repetições. As fontes de $\mathrm{P}$ foram fosfato monopotássico e ácido fosfórico, implicando no uso de nitrato de sódio, cloreto de cálcio e cloreto de potássio, nos tratamentos 1 e 2 ; 3 e 4; e 2 e 4, respectivamente, para balancear as soluções. As concentrações de Na na fase de crescimento vegetativo nos tratamentos 1 e 2 foram de $1,7 \mathrm{mmol} \mathrm{L}^{-1}\left(39,2 \mathrm{mg} \mathrm{L}^{-1}\right)$. O $\mathrm{Cl}$ apresentou-se nas concentrações de $0,3 \mathrm{mmol} \mathrm{L}^{-1}\left(10,7 \mathrm{mg} \mathrm{L}^{-1}\right)$ na fase vegetativa dos tratamentos 3 e 4 , e na concentração de $3,4 \mathrm{mmol} \mathrm{L}^{-1}$ (115,2 mg $\mathrm{L}^{-1}$ ) na fase de frutificação dos tratamentos 2 e 4 .

Foi utilizado o tomate híbrido Carmen, longa vida, plurilocular, tipo salada, de crescimento indeterminado. As sementes foram semeadas em copos plásticos de $50 \mathrm{ml}$ contendo vermiculita. Do semeio ao $10^{\circ}$ dia, foram irrigadas com água desionizada; do $10^{\circ}$ ao $14^{\circ}$ dia, com solução de Steiner a $1 / 2$ força iônica e do $15^{\circ}$ ao transplante, irrigadas com a solução de Steiner a 1 força iônica (Steiner, 1984). Todas as irrigações foram realizadas uma vez ao dia, após as 18 horas, em volume suficiente para saturar o substrato. Aos 36 dias, quando as plantas atingiram de 10 a $15 \mathrm{~cm}$ de altura, foram selecionadas e transplantadas para vasos plásticos de $8,6 \mathrm{~L}$, com espaçamento de $0,35 \mathrm{~m}$ entre plantas e de $0,70 \mathrm{~m}$ entre linhas, contendo as soluções nutritivas que foram calculadas com base nos trabalhos de Adams (1994). A partir dessa data, mediram-se diariamente as temperaturas no interior da estufa, por termômetro colocado fora de abrigo, à altura de $1,10 \mathrm{~m}$ da superfície do piso.

$\mathrm{O}$ pH da solução em todos os tratamentos foi monitorado e ajustado à faixa de 5,5 a 6,5 utilizando-se $\mathrm{HCl}$ ou $\mathrm{NaOH}$. Foram realizadas reposições periódicas das soluções, com base na redução da condutividade elétrica admitindo-se até 30\% de depleção. Entre essas reposições, o volume da solução de cada vaso era medido e completado com água deionizada admitindo-se uma redução máxima de $40 \%$ do seu volume inicial.

As plantas foram conduzidas com um cacho, sendo a poda apical realizada aos 20 dias após o transplantio
(DAT), deixando-se 3 folhas acima do primeiro cacho, conforme preconizado por Morgan (1996) e Morgan (1997a). Aos 30 DAT foram trocadas as soluções iniciais de crescimento pelas soluções de frutificação. Aos 42 DAT foi feito o desbaste dos frutos, deixando seis frutos em cada cacho. Durante a condução do experimento foram necessárias podas periódicas para manutenção do número de frutos e folhas preconizados.

Para sustentar as plantas utilizaramse tampas de isopor revestidas com papel alumínio. As tampas tinham um orifício central para o encaixe de espuma que serviu de suporte e proteção para a planta. A oxigenação das soluções nutritivas foi feita por compressor de ar.

Houve ocorrência de oídio, sendo necessária a aplicação de fungicida (Thiovit Br, 2g L-1) nos dias 30/06 e 26/ 07/99.

Os frutos foram coletados para análise quando atingiram a completa coloração vermelha. A colheita iniciou-se aos 80 DAT e terminou aos 96 DAT. Os frutos foram classificados de acordo com a portaria do Ministério da Agricultura e Reforma Agrária [Gigante com maior diâmetro transversal (MDT) > $100 \mathrm{~mm}$; Grande com MDT entre $80 \mathrm{e}$ $100 \mathrm{~mm}$; Médio com MDT entre 65 e $80 \mathrm{~mm}$ e Pequeno com MDT entre $50 \mathrm{e}$ $65 \mathrm{~mm}$ ]. Avaliou-se no primeiro fruto colhido o peso da matéria fresca, o diâmetro e a espessura do pericarpo, os sólidos solúveis totais, a acidez titulável, o pH (Instituto Adolfo Lutz, 1985) e os teores de licopeno e de carotenóides totais (Zscheile \& Porter, 1947). No segundo fruto, foram avaliados os pesos das matérias fresca e seca, o diâmetro e a espessura do pericarpo e os teores de nutrientes ( $\mathrm{Ca}, \mathrm{Mg}$ e $\mathrm{K}$ ). Para análise do teor de nutrientes, o fruto foi dividido transversalmente em três porções: proximal, mediana e distal, em relação ao pedúnculo. Nessa característica os dados obtidos foram analisados em parcelas subdivididas, sendo as parcelas compostas pelas soluções nutritivas e as subparcelas pelas porções dos frutos. Nos demais frutos foram avaliados o peso da matéria fresca, o diâmetro e a espessura do pericarpo.

Foi realizada uma amostragem para diagnose foliar retirando-se a primeira 
Tabela 1. Conjuntos de fontes de nutrientes componentes das soluções nutritivas utilizadas nas fases de crescimento vegetativo e de frutificação do tomateiro. Viçosa, UFV, 1999.

\begin{tabular}{|c|c|c|c|c|c|c|c|}
\hline \multicolumn{4}{|c|}{ Crescimento Vegetativo } & \multicolumn{4}{|c|}{ Frutificação } \\
\hline Conj. A & g 1000L-1 & Conj. B & g $1000 L^{-1}$ & Conj. C & g $1000 \mathrm{~L}^{-1}$ & Conj. D & g $1000 \mathrm{~L}^{-1}$ \\
\hline $\mathrm{KH}_{2} \mathrm{PO}_{4}$ & 272,0 & $\mathrm{H}_{3} \mathrm{PO}_{4}$ & 115,8 & $\mathrm{KH}_{2} \mathrm{PO}_{4}$ & 408,7 & $\mathrm{H}_{3} \mathrm{PO}_{4}$ & 173,7 \\
\hline $\mathrm{MgSO}_{4}$ & 246,4 & $\mathrm{MgSO}_{4}$ & 246,4 & $\mathrm{MgSO}_{4}$ & 369,6 & $\mathrm{MgSO}_{4}$ & 369,6 \\
\hline $\mathrm{KNO}_{3}$ & 213,6 & $\mathrm{KNO}_{3}$ & 427,3 & $\mathrm{KNO}_{3}$ & 597,0 & $\mathrm{KNO}_{3}$ & 587,8 \\
\hline $\mathrm{Ca}\left(\mathrm{NO}_{3}\right)_{2}$ & 421,0 & $\mathrm{Ca}\left(\mathrm{NO}_{3}\right)_{2}$ & 389,3 & $\mathrm{Ca}\left(\mathrm{NO}_{3}\right)_{2}$ & 631,6 & $\mathrm{Ca}\left(\mathrm{NO}_{3}\right)_{2}$ & 631,6 \\
\hline $\mathrm{NaNO}_{3}$ & 144,9 & $\mathrm{CaCl}_{2}$ & 33,1 & & & $\mathrm{KCl}$ & 241,7 \\
\hline $\mathrm{FeCl}_{3}$ & 9,6 & $\mathrm{FeCl}_{3}$ & 9,6 & $\mathrm{FeCl}_{3}$ & 15,9 & $\mathrm{FeCl}_{3}$ & 15,9 \\
\hline $\mathrm{Na}_{2}$-EDTA & 13,2 & $\mathrm{Na}_{2}$-EDTA & 13,2 & $\mathrm{Na}_{2}$-EDTA & 20,0 & $\mathrm{Na}_{2}$-EDTA & 20,0 \\
\hline $\mathrm{MnSO}_{4}$ & 3,7 & $\mathrm{MnSO}_{4}$ & 3,7 & $\mathrm{MnSO}_{4}$ & 5,5 & $\mathrm{MnSO}_{4}$ & 5,5 \\
\hline $\mathrm{H}_{3} \mathrm{BO}_{3}$ & 1,3 & $\mathrm{H}_{3} \mathrm{BO}_{3}$ & 1,3 & $\mathrm{H}_{3} \mathrm{BO}_{3}$ & 1,9 & $\mathrm{H}_{3} \mathrm{BO}_{3}$ & 1,9 \\
\hline $\mathrm{ZnSO}_{4}$ & 1,3 & $\mathrm{ZnSO}_{4}$ & 1,3 & $\mathrm{ZnSO}_{4}$ & 1,3 & $\mathrm{ZnSO}_{4}$ & 1,3 \\
\hline $\mathrm{Na}_{2} \mathrm{MoO}_{4}$ & 0,2 & $\mathrm{Na}_{2} \mathrm{MoO}_{4}$ & 0,2 & $\mathrm{Na}_{2} \mathrm{MoO}_{4}$ & 0,2 & $\mathrm{Na}_{2} \mathrm{MoO}_{4}$ & 0,2 \\
\hline $\mathrm{CuSO}_{4}$ & 0,1 & $\mathrm{CuSO}_{4}$ & 0,1 & $\mathrm{CuSO}_{4}$ & 0,2 & $\mathrm{CuSO}_{4}$ & 0,2 \\
\hline
\end{tabular}

folha imediatamente abaixo do primeiro cacho, no momento da colheita do primeiro fruto maduro.

Para a análise química, o material amostrado foi lavado em água deionizada e seco em estufa com circulação forçada de ar a $70^{\circ} \mathrm{C}$, por 72 horas. Em seguida, foi pesado para determinar o teor de matéria seca e moído em moinho tipo Wiley equipado com peneira de 20 mesh. O N-total foi determinado pelo método Kjeldahl descrito por Bremner (1965). Os elementos P, K, $\mathrm{Ca}, \mathrm{Mg}, \mathrm{S}, \mathrm{Fe}, \mathrm{Mn}, \mathrm{Zn}$ e $\mathrm{Cu}$ foram analisados após a mineralização pela digestão nítrico-perclórica. O B foi determinado colorimetricamente pelo método da Azometina H (Wolf, 1974), após a mineralização por via seca em mufla a $550^{\circ} \mathrm{C}$. O P foi dosado colorimetricamente, pelo método de redução do fosfomolibdato pela vitamina C conforme Braga \& Defelipo (1974); o K, por fotometria de emissão de chama; o $\mathrm{Ca}, \mathrm{Mg}$, Fe, $\mathrm{Mn}, \mathrm{Zn}$ e $\mathrm{Cu}$, por espectrofotometria de absorção atômica e o S, determinado por turbidimetria do sulfato (Blanchar et al., 1965). Os dados obtidos foram submetidos à análise de variância e os efeitos dos tratamentos comparados pelo teste de Tukey a $5 \%$ de probabilidade.

\section{RESULTADOS E DISCUSSÃO}

Para os conjuntos de fontes de nutrientes A e B utilizados na fase de cres- cimento vegetativo, obtiveram-se inicialmente os valores de 1,2 e 1,1 mS $\mathrm{cm}^{-1}$ para a condutividade elétrica (CE) e valores de $\mathrm{pH}$ de 6,0 e 2,9, respectivamente. Apesar de os valores da condutividade elétrica estarem abaixo de $2 \mathrm{mS} \mathrm{cm}^{-1}$, indicados por Morgan (1996) e Morgan (1997a), como adequado para tomateiro com um cacho, as soluções proporcionaram crescimento satisfatório às plantas, verificado pela alta demanda de podas nessa fase do experimento. Para os conjuntos de fontes C e D utilizados na fase de frutificação, obtiveram-se inicialmente os valores de 1,8 e $2,5 \mathrm{mS} \mathrm{cm}^{-1}$, para a $\mathrm{CE}$ e de 5,6 e 2,7 para $\mathrm{pH}$, respectivamente. As soluções tiveram os valores de $\mathrm{pH}$ corrigidos para a faixa de 5,5 a 6,5 (Martinez, 1999), antes de serem fornecidas às plantas.

O consumo de água pelas plantas de tomate foi mais acentuado no período de frutificação, atingindo o máximo de seis litros por planta por semana. A variação no consumo ocorreu em função da condutividade elétrica, sendo os maiores consumos totais observados para os tratamentos compostos pelos conjuntos de fontes de nutrientes com menor condutividade elétrica.

Para as características produção por planta, peso e diâmetro dos frutos, espessura do pericarpo, $\mathrm{pH}$ do pericarpo e da polpa, matéria seca dos frutos, folíolos e pecíolos, ácido cítrico no pericarpo e na polpa, sólidos solúveis no pericarpo e na polpa, carotenóides totais e licopeno não foram encontradas diferenças significativas entre os tratamentos (Tabela 2), o que indica que concentrações de $39,2 \mathrm{mg} \mathrm{L}^{-1}\left(1,7 \mathrm{mmol} \mathrm{L}^{-1}\right)$ de $\mathrm{Na}^{+}$na fase de crescimento vegetativo e $115,2 \mathrm{mg} \mathrm{L}^{-1}\left(3,4 \mathrm{mmol} \mathrm{L}^{-1}\right)$ de $\mathrm{Cl}^{-}$na fase de frutificação não foram prejudiciais ao cultivo de tomates híbrido Carmen. Kafkafi et al. (1984) relatam que o tomateiro pode ser cultivado na presença de concentrações de $\mathrm{Cl}^{-}$de até $10 \mathrm{mmo} \mathrm{L}^{-1}$, desde que as concentrações de $\mathrm{NO}_{3}^{-}$sejam mantidas elevadas.

Durante o experimento as temperaturas médias semanais apresentaram mínima entre 4,2 e $10^{\circ} \mathrm{C}$ e máxima entre 23,3 e $34,4^{\circ} \mathrm{C}$. Para cada fase do ciclo do tomateiro existe temperatura considerada ideal, mas, de maneira geral, temperaturas noturnas entre 15 e $20^{\circ} \mathrm{C} \mathrm{e}$ diurnas entre 25 e $30^{\circ} \mathrm{C}$ são consideradas como favoráveis para seu bom desenvolvimento e produção (Brandão Filho \& Callegari, 1999).

A produção média por planta foi de 920 g (Tabela 2), com um ciclo de 96 DAT à última colheita, o que no espaçamento adotado corresponderia a uma produtividade média de $142 \mathrm{t} \mathrm{ha}^{-1}$ ano $^{-1}$. Entretanto, as baixas temperaturas mínimas observadas durante o período de condução do experimento podem ter afetado negativamente os resultados. 
Tabela 2. Características da planta e do fruto de tomate longa vida, híbrido Carmen, cultivados em hidroponia em função dos tratamentos nutricionais. Viçosa, UFV, 1999.

\begin{tabular}{|c|c|c|c|c|c|c|}
\hline \multirow{2}{*}{ Características } & \multicolumn{4}{|c|}{ Tratamentos } & \multirow{2}{*}{ Média } & \multirow{2}{*}{$\mathrm{CV}(\%)$} \\
\hline & $1^{1 /}$ & $2^{21}$ & $3^{3 /}$ & $4^{4 /}$ & & \\
\hline Produção por planta (g) & $960,0 \mathrm{~A}$ & $929,3 \mathrm{~A}$ & $954,1 \mathrm{~A}$ & $835,4 \mathrm{~A}$ & 919,7 & 20,09 \\
\hline Peso dos frutos (g) & $160,0 \mathrm{~A}$ & $154,9 \mathrm{~A}$ & $160,7 \mathrm{~A}$ & $143,1 \mathrm{~A}$ & 154,7 & 17,51 \\
\hline Diâmetro do fruto $(\mathrm{cm})$ & $7,1 \mathrm{~A}$ & $7,1 \mathrm{~A}$ & $7,2 \mathrm{~A}$ & $7,0 \mathrm{~A}$ & 7,1 & 6,86 \\
\hline Espessura do pericarpo $(\mathrm{cm})$ & $1,0 \mathrm{~A}$ & $1,0 \mathrm{~A}$ & $1,0 \mathrm{~A}$ & $0,9 \mathrm{~A}$ & 1,0 & 6,92 \\
\hline Acido cítrico no pericarpo(\%) & $0,4 \mathrm{~A}$ & $0,4 \mathrm{~A}$ & $0,4 \mathrm{~A}$ & $0,4 \mathrm{~A}$ & 0,4 & 11,80 \\
\hline Acido cítrico na polpa (\%) & $0,5 \mathrm{~A}$ & $0,6 \mathrm{~A}$ & $0,6 \mathrm{~A}$ & $0,6 \mathrm{~A}$ & 0,6 & 14,93 \\
\hline Sólidos solúveis do pericarpo (dag kg-1) & $5,4 \mathrm{~A}$ & $5,0 \mathrm{~A}$ & $5,1 \mathrm{~A}$ & $5,2 \mathrm{~A}$ & 5,1 & 10,30 \\
\hline Sólidos solúveis da polpa (dag kg-1) & $5,2 \mathrm{~A}$ & $5,0 \mathrm{~A}$ & $4,9 \mathrm{~A}$ & $4,8 \mathrm{~A}$ & 5,0 & 6,81 \\
\hline Carotenóides totais (mg g-1) & $289,0 \mathrm{~A}$ & $260,1 \mathrm{~A}$ & $259,1 \mathrm{~A}$ & $246,3 \mathrm{~A}$ & 263,6 & 18,50 \\
\hline Licopeno (mg g-1) & $116,5 \mathrm{~A}$ & $104,4 \mathrm{~A}$ & $101,2 \mathrm{~A}$ & $99,6 \mathrm{~A}$ & 105,4 & 18,63 \\
\hline pH do pericarpo & $4,3 \mathrm{~A}$ & $4,2 \mathrm{~A}$ & $4,2 \mathrm{~A}$ & $4,2 \mathrm{~A}$ & 4,21 & 3,20 \\
\hline $\mathrm{pH}$ da polpa & $4,2 \mathrm{~A}$ & $4,1 \mathrm{~A}$ & $4,1 \mathrm{~A}$ & $4,1 \mathrm{~A}$ & 4,1 & 3,70 \\
\hline Matéria seca dos frutos (\%) & $5,8 \mathrm{~A}$ & $5,6 \mathrm{~A}$ & $5,6 \mathrm{~A}$ & $5,7 \mathrm{~A}$ & 5,7 & 7,00 \\
\hline Matéria seca dos folíolos (\%) & $12,3 \mathrm{~A}$ & $10,9 \mathrm{~A}$ & $12,1 \mathrm{~A}$ & $10,6 \mathrm{~A}$ & 11,5 & 12,32 \\
\hline Matéria seca dos pecíolos(\%) & $10,2 \mathrm{~A}$ & $9,3 \mathrm{~A}$ & $9,5 \mathrm{~A}$ & $9,0 \mathrm{~A}$ & 9,5 & 10,80 \\
\hline
\end{tabular}

${ }^{1 /}$ Conjuntos $\mathrm{A}+\mathrm{C}$ da tabela $1 ;{ }^{2 /}$ Conjuntos $\mathrm{A}+\mathrm{D}$ da tabela $1 ;{ }^{3 /}$ Conjuntos $\mathrm{B}+\mathrm{C}$ da tabela $1 ;{ }^{4 /}$ Conjuntos $\mathrm{B}+\mathrm{D}$ da tabela 1 ;

Em cada linha, as médias seguidas de pelo menos uma mesma letra não diferem entre si, a $5 \%$ de probabilidade, pelo teste de Tukey.

De acordo com Adams et al. (2001), baixas temperaturas podem influenciar a velocidade de crescimento dos frutos e o ciclo da cultura. Quando plantas de tomateiro foram cultivadas sob condições de temperaturas controladas de 14 , 18,22 e $26^{\circ} \mathrm{C}$ a maturação dos frutos ocorreu com 95, 65, 46 e 42 dias após a abertura das flores, respectivamente. Além do aumento do ciclo com a diminuição da temperatura, foram obtidos também menor peso médio de frutos e menor produção por planta. Morgan (1996) obteve de 420 a 500 g planta $^{-1}$ e ciclo médio de 80 a 85 dias no inverno e 60 dias no verão, com média de três variedades de tomate cultivadas na densidade de 13,5 plantas $\mathrm{m}^{-2}$ e CE da solução nutritiva variando de 2 a $8 \mathrm{mS}$ $\mathrm{cm}^{-1}$ na fase de frutificação, deixando seis frutos por cacho. Quanto ao ciclo de cultivo obtido neste experimento é interessante salientar que comercialmente os frutos de tomate são colhidos a partir do momento que atingem a maturidade fisiológica e neste trabalho foram colhidos plenamente maduros, o que também contribuiu para um maior ciclo.

As plantas produziram frutos com $154,7 \mathrm{~g}$ de peso médio, $7,1 \mathrm{~cm}$ de diâmetro e $1,0 \mathrm{~cm}$ de espessura do pericarpo (Tabela 2). De acordo com a classificação do Ministério da Agricultura e Reforma Agrária foram produzidos $25,4 \%$ de frutos pequenos, $60 \%$ de frutos médios e $14,6 \%$ de frutos grandes. Os frutos grandes representaram $29,2 \%$ da produção total. Camargos (1998), trabalhando com o híbrido Carmen em solo sob estufa e fertirrigação, variando espaçamento e número de cachos, obteve que a participação de frutos grandes na produção total variou de 5,8 a 37,4\%. Segundo a classificação da União Européia, os frutos poderiam ser considerados grandes e muito grandes. A tendência atual é valorizar mais a qualidade e sabor que tendem a ser melhores em frutos menores, em função da menor absorção de água e maior concentração de ácidos e açúcares. Logendra \& Janes (1999), em cultivo de tomateiro com apenas um cacho, no espaçamento de $0,3 \times 0,3 \mathrm{~m}$, obtiveram frutos com peso médio de $225 \mathrm{~g}$ e produção de $1.090 \mathrm{~g}$ planta $^{-1}$, porém esses valores foram obtidos de 4,8 frutos por planta e com outra variedade.

De acordo com Morgan (1997b), tem havido preocupação crescente com o aumento da qualidade dos frutos de tomate. $\mathrm{O}$ autor discute que ao longo do tempo a qualidade reduziu-se devido ao melhoramento voltado para altas produtividades e aumento da vida de prateleira. Os principais componentes da qualidade seriam matéria seca, firmeza da polpa, vida de prateleira, teor de açúcares e teor de ácidos. Winsor (1966) afirma que o paladar do tomate é dado pelo adequado balanço entre açúcares e ácidos. Frutos com muito açúcar e sem acidez são insípidos, muita acidez e pouco açúcar levam a um sabor acre, pouca acidez e pouco açúcar resulta em sabor aguado.

O teor de ácido cítrico na polpa $(0,6 \%)$ foi maior que o teor no pericarpo $(0,4 \%)$ (Tabela 2) à semelhança dos resultados de Rêgo (1997), sendo que esses valores foram menores que os obtidos por Camargos (1998). O sabor do fruto de tomate é melhor próximo ao estádio vermelho-laranja, quando o conteúdo de açúcar é alto, antes do decréscimo da acidez que é elevada no estádio verde maduro e diminui com o amadurecimento (Morgan, 1997b).

Os teores de sólidos solúveis obtidos estão de acordo com Sampaio (1996) e Rêgo (1997), que trabalharam com a cv. Santa Clara, cultivada em solo. Porém, os valores de 5,0 e 5,1 dag kg-1 
Tabela 3. Concentrações de macro e micronutrientes em folhas de plantas de tomate longa vida, híbrido Carmen, cultivadas em hidroponia em função dos tratamentos nutricionais. Viçosa, UFV, 1999.

\begin{tabular}{lccccccccccc}
\hline \multirow{2}{*}{ Tratam. } & $\mathbf{N}$ & $\mathbf{P}$ & $\mathbf{K}$ & $\mathbf{C a}$ & $\mathbf{M g}$ & $\mathbf{S}$ & $\mathbf{F e}$ & $\mathbf{Z n}$ & $\mathbf{C u}$ & $\mathbf{B}$ & $\mathbf{M n}$ \\
\hline & \multicolumn{9}{c}{$\left.\mathbf{( d a g ~ k g}^{-1}\right)$} \\
\hline $1^{1 /}$ & $3,3 a$ & $1,1 \mathrm{a}$ & $4,7 a$ & $4,1 a$ & $0,9 a$ & $1,7 a$ & $198 a$ & $88 a$ & $8 a$ & $206 a$ & $603 a$ \\
$2^{2 /}$ & $3,2 a$ & $1,3 a$ & $5,4 a$ & $4,6 a$ & $0,9 a$ & $1,8 a$ & $220 a$ & $104 a$ & $10 a$ & $215 a$ & $694 a$ \\
$3^{3 /}$ & $3,0 a$ & $1,3 a$ & $5,0 a$ & $4,7 a$ & $0,9 a$ & $1,8 a$ & $206 a$ & $97 a$ & $9 a$ & $207 a$ & $676 a$ \\
$4^{4 /}$ & $3,2 a$ & $1,3 a$ & $5,4 a$ & $4,6 a$ & $0,8 a$ & $1,9 a$ & $213 a$ & $95 a$ & $11 a$ & $206 a$ & $685 a$ \\
Média & 3,2 & 1,3 & 5,1 & 4,5 & 0,9 & 1,8 & 209 & 96 & 10 & 209 & 665 \\
\hline CV $(\%)$ & 27,2 & 16,3 & 13,5 & 15,9 & 12,9 & 14,3 & 14,0 & 20,6 & 24,2 & 10,5 & 17,1 \\
\hline
\end{tabular}

${ }^{1 /}$ Conjuntos $\mathrm{A}+\mathrm{C}$ da tabela $1 ;{ }^{2 /}$ Conjuntos $\mathrm{A}+\mathrm{D}$ da tabela $1 ;{ }^{3 /}$ Conjuntos $\mathrm{B}+\mathrm{C}$ da tabela $1 ;{ }^{4 /}$ Conjuntos $\mathrm{B}+\mathrm{D}$ da tabela 1 ;

Em cada linha, as médias seguidas de pelo menos uma mesma letra não diferem entre si, a 5\% de probabilidade, pelo teste de Tukey.

Tabela 4. Concentrações de $\mathrm{Ca}, \mathrm{Mg}$ e $\mathrm{K}$ nas porções proximal, mediana e distal de frutos de tomate longa vida, híbrido Carmen, cultivados em hidroponia em função dos tratamentos nutricionais. Viçosa, UFV, 1999.

\begin{tabular}{|c|c|c|c|c|c|}
\hline & \multirow{3}{*}{ Porção } & \multicolumn{4}{|c|}{ Tratamentos } \\
\hline & & $1^{1 /}$ & $2^{21}$ & $3^{3 /}$ & $4^{4 \prime}$ \\
\hline & & \multicolumn{4}{|c|}{$\left(\right.$ dag kg $\left.^{-1}\right)$} \\
\hline & proximal & $0,1 \mathrm{Aa}$ & $0,2 \mathrm{Aa}$ & $0,2 \mathrm{Aa}$ & $0,1 \mathrm{Aa}$ \\
\hline \multirow[t]{3}{*}{$\mathrm{Ca}$} & mediana & $0,1 \mathrm{Ab}$ & $0,1 \mathrm{Ab}$ & $0,1 \mathrm{Ab}$ & $0,1 \mathrm{Ab}$ \\
\hline & distal & $0,1 \mathrm{Ab}$ & $0,1 \mathrm{Ab}$ & $0,1 \mathrm{Ab}$ & $0,1 \mathrm{Ab}$ \\
\hline & proximal & $0,2 A b$ & $0,2 A b$ & $0,2 A b$ & $0,2 \mathrm{Ab}$ \\
\hline \multirow[t]{3}{*}{$\mathrm{Mg}$} & mediana & $0,2 \mathrm{Aa}$ & $0,2 \mathrm{Aa}$ & $0,2 \mathrm{Aa}$ & $0,2 \mathrm{Aa}$ \\
\hline & distal & $0,2 \mathrm{Aa}$ & $0,2 \mathrm{Aa}$ & $0,2 \mathrm{Aa}$ & $0,2 \mathrm{Aa}$ \\
\hline & proximal & $5,5 \mathrm{Aa}$ & $5,5 \mathrm{Aa}$ & $5,5 \mathrm{Aa}$ & $5,6 \mathrm{Aa}$ \\
\hline \multirow[t]{2}{*}{ K } & mediana & $5,3 \mathrm{Aa}$ & 5,33Aa & $5,1 \mathrm{Ab}$ & $5,2 \mathrm{Ab}$ \\
\hline & distal & $5,2 \mathrm{Aa}$ & $5,4 \mathrm{Aa}$ & 5,3Aab & $5,4 \mathrm{Aab}$ \\
\hline
\end{tabular}

${ }^{1 /}$ Conjuntos $\mathrm{A}+\mathrm{C}$ da tabela $1 ;{ }^{2 /}$ Conjuntos $\mathrm{A}+\mathrm{D}$ da tabela $1 ;{ }^{3 /}$ Conjuntos $\mathrm{B}+\mathrm{C}$ da tabela $1 ;{ }^{4 /}$ Conjuntos $\mathrm{B}+\mathrm{D}$ da tabela 1 ;

Em cada linha, as médias seguidas de pelo menos uma mesma letra não diferem entre si, a 5\% de probabilidade, pelo teste de Tukey.

para os teores de sólidos solúveis na polpa e pericarpo, respectivamente (Tabela 2), são superiores ao teor médio de 4,5 dag $\mathrm{kg}^{-1}$ encontrado por Camargos (1998) para tomate em cultivo protegido, em solo. Segundo Morgan (1997c), valores de grau Brix acima de 5 indicam frutos de tomate de alta qualidade. $\mathrm{O}$ aumento do teor de sólidos solúveis está relacionado com o sabor dos frutos, por ser essa fração composta por ácidos e açúcares, conferindo assim maior qualidade ao produto.

O teor de licopeno $\left(105,4 \mathrm{mg} \mathrm{g}^{-1}\right)$ foi semelhante ao valor de $105,7 \mathrm{mg} \mathrm{g}^{-1}$ encontrado por Rêgo (1997), em frutos maduros do cv. Santa Clara, porém acima do valor de $87,3 \mathrm{mg} \mathrm{g}^{-1}$ encontrado por Camargos (1998), que também trabalhou com o híbrido Carmen. Para os carotenóides totais obtiveram-se $263,6 \mathrm{mg}$ $\mathrm{g}^{-1}$ em comparação a $119,8 \mathrm{mg} \mathrm{g}^{-1}$ encontrado por Camargos (1998) (Tabela 2).

Os valores de $\mathrm{pH}$ de 4,1 e 4,2 para polpa e pericarpo, respectivamente, e de matéria seca dos frutos (MSF) de 5,7\% encontrados (Tabela 4) são semelhantes aos valores obtidos por Rêgo (1997) e Camargos (1998). Morgan (1997c) considera que valores de matéria seca dos frutos são normais dentro da faixa de 4 a $6 \%$. Para a matéria seca dos folíolos e pecíolos foram obtidos $11,5 \%$ e 9,5\%, respectivamente (Tabela 2 ).

Os teores de nutrientes nos tecidos não foram influenciados pelos tratamentos (Tabela 3). Os teores de $\mathrm{Mg}, \mathrm{Cu}, \mathrm{Zn}$ e Fe observados estão dentro da faixa considerada adequada, o $\mathrm{N}$ ficou abaixo dessa faixa e o P, K, S, Ca, Mn e B se encontram acima, considerando-se os valores de referência citados por Reuter \&
Robinson (1988). Fayad (1998) encontrou na matéria seca de folhas do tomateiro, no início da colheita, os valores de 3,$8 ; 0,3 ; 2,9 ; 5,3 ; 0,8 ; \mathrm{e} 1,6 \mathrm{dag} \mathrm{kg}^{-1}$ para $\mathrm{N}, \mathrm{P}, \mathrm{K}, \mathrm{Ca}, \mathrm{Mg}$ e S, respectivamente, e 175; 543; 323; e $1.282 \mathrm{mg} \mathrm{kg}^{-1}$ para $\mathrm{Zn}$, $\mathrm{Fe}, \mathrm{Cu}$ e $\mathrm{Mn}$, respectivamente.

As concentrações de $\mathrm{N}$ das soluções empregadas, tanto na fase de crescimento vegetativo quanto na fase de frutificação, estão na faixa adequada quando comparadas a recomendada por Grobler (1999), citado por Lee (1999), para o cultivo de tomates sob fertirrigação. Kafkafi et al. (1984) relataram que concentrações de $\mathrm{N}$ na faixa de $10 \pm 3 \mathrm{mmol} \mathrm{L}^{-1}$ são adequadas para o cultivo do tomate, devendo-se empregar concentrações maiores em presença de concentrações elevadas de $\mathrm{Cl}$ e sob alta intensidade luminosa. 
As concentrações de P e K empregadas neste trabalho são maiores quando comparadas às preconizadas por Grobler (1999), citado por Lee (1999), justificando os elevados teores desses elementos observados nos tecidos (Tabela 3). As concentrações de $\mathrm{P}$ e K apresentadas por Martinez (1997), Martinez et al. (1997) e por Furlani et al. (1999b), para tomate, mostram variações entre as recomendações, porém esses autores têm em comum o uso de altas concentrações desses elementos.

A relação $\mathrm{N}: \mathrm{K}$ na fase de frutificação foi de 1:2, o que está de acordo com o proposto por Adams \& Massey (1984) e Adams (1994). Segundo esses autores, nas primeiras semanas de cultivo, a absorção de $\mathrm{N}$ e K ocorre na proporção de 1,0:1,2, porém com o aparecimento dos frutos se eleva até valores de 1,0:2,5, reduzindo-se posteriormente para 1,0:2,0. Grobler (1999), citado por Lee (1999), recomenda a relação 5: 3: 8 de $\mathrm{N}: \mathrm{P}: \mathrm{K}$ para obter-se boa qualidade de tomates. No presente trabalho essa relação foi de 5,4:3:10,8.

Embora as concentrações nos tecidos tenham se apresentado adequadas para $\mathrm{Mg}$ e elevadas para $\mathrm{Ca}$ e $\mathrm{S}$, as concentrações desses nutrientes na solução nutritiva estão no limite inferior das recomendações de diversos autores para tomate (Martinez, 1997; Martinez et al., 1997; Furlani et al., 1999b), especialmente a de $\mathrm{S}$ que é considerado um nutriente bastante exigido pelo tomateiro (Cerdá et al., 1984). É possível que para o cultivo em outras épocas do ano, que propiciam maior velocidade de crescimento, as concentrações desses nutrientes na solução nutritiva necessitem de reajuste.

As concentrações de micronutrientes empregadas na solução nutritiva estão dentro da faixa preconizada para o cultivo hidropônico do tomateiro por diversos autores (Martinez, 1997, Martinez et al., 1997), podendo as concentrações de $\mathrm{B}$ e Fe serem consideradas relativamente baixas e a de Mn mediana, não justificando os teores foliares elevados de $\mathrm{B}$ e $\mathrm{Mn}$. O valor médio de Mn observado foi de $665 \mathrm{mg} \mathrm{kg}^{-1}$ (Tabela 3), segundo Reuter \& Robinson (1988), para folhas nesse estádio, valores acima de $100 \mathrm{mg} \mathrm{kg}^{-1}$ já são considerados eleva- dos. Mourão (1978) relata que níveis mais altos de Mn em tomate foram tóxicos apenas para as plantas submetidas à deficiência de B. Plantas de tomate foram mais tolerantes que outras plantas a concentrações internas de Mn nos trabalhos de Dennis (1968) e Foy et al. (1978), citados por El-Jaoual \& Cox (1998). Uma possível justificativa para as elevadas concentrações foliares desses elementos seria sua presença como contaminantes em alguns dos adubos utilizados no preparo das soluções nutritivas.

A partição de $\mathrm{Ca}, \mathrm{Mg}$ e $\mathrm{K}$ nos frutos de tomate não foi influenciada pelas soluções nutritivas, entretanto observaram-se variações em função da porção do fruto. $\mathrm{O} \mathrm{Ca}$ e $\mathrm{K}$ apresentaram maior concentração na região proximal, ao contrário do Mg, que nessa porção, apresentou menor concentração (Tabela 4). Comportamento semelhante para o $\mathrm{Ca}$, de menores concentrações na região distal, foi observado por Castellane (1985). Segundo Wien (1997), este comportamento ocorre em função do desequilíbrio entre o rápido crescimento celular do fruto e a baixa taxa de aquisição de $\mathrm{Ca}$, principalmente pela região distal. Adams \& Ho (1995) obtiveram concentração de $\mathrm{Ca}$ em frutos de tomate aproximadamente seis vezes menor que a dos frutos de pepino. Essa diferença, segundo os autores, é refletida pela maior densidade de estômatos no fruto de pepino, sugerindo que a transpiração do fruto tem importante papel na concentração de $\mathrm{Ca}$ nos diferentes órgãos da planta. Embora a concentração de Ca da solução nutritiva não tenha sido elevada, não se observou a ocorrência de fundo preto, o que pode ser devido às temperaturas amenas, que levaram a um crescimento menos acentuado dos frutos.

As concentrações médias nos frutos dos nutrientes $\mathrm{Ca}, \mathrm{Mg}$ e $\mathrm{K}$, obtidas nas três porções (Tabela 4), foram 0,$1 ; 0,2$; e $5,4 \mathrm{dag} \mathrm{kg}^{-1}$, respectivamente. Gertsson (1995), trabalhando também com tomate em hidroponia, obteve concentrações de 0,$1 ; 0,2 ;$ e $4,7 \mathrm{dag} \mathrm{kg}^{-1}$, para Ca, $\mathrm{Mg}$ e $\mathrm{K}$, respectivamente. A concentração de $\mathrm{K}$ nos frutos foi maior do que a concentração média nas folhas. Takahashi (1989) encontrou que o K representa $80 \%$ do total de cátions acumulados no fruto. Segundo Ho \& Adams (1995), o Ké acumulado em grandes quantidades nos frutos de tomate e é requerido para uniformizar o amadurecimento e aumentar a acidez do fruto, características essenciais para boa qualidade e sabor.

\section{AGRADECIMENTOS}

Agradecemos à UFV pela oportunidade de realização do trabalho e à CAPES pela concessão da bolsa de pesquisa.

\section{LITERATURA CITADA}

ADAMS, P.; MASSEY, D.M. Nutrient uptake by tomatoes from recirclating solutions. In: INTERNATIONAL CONGRESS ON SOILLESS CULTURE, 6., 1984, Lunteren. Proceedings...Lunteren: International Society of Soiless Culture, 1984. p. 71-79.

ADAMS, P. Nutrition of greenhouse vegetables in NFT and hydroponic systems. Acta Horticulturae, n. 361, p. 245-257, 1994.

ADAMS, P.; HO, L.C. Differential effects of salinity and humidity on growth and $\mathrm{Ca}$ status of tomato and cucumber grown in hydroponic culture. In: GROWING MEDIA E PLANT NUTRITION. Acta Horticulturae, n. 401, p. $357-$ $363,1995$.

ADAMS, S.R.; COCKSHULL, K. E.; CAVE, R.J. Effect of temperature on the growth and development of tomato fruits. Annals of Botany, n. 88 , p. 869-877, 2001.

BLANCHAR, R.W.; REHM, G.; CALDWELL, A.C. Sulfur in plant material by digestion with nitric and perchloric acid. Proceedings-Soil Science Society of America, v. 29, n. 1, p. 71-72, 1965.

BRAGA, J.M.; DEFELIPO, B. Determinação espectrofotométrica de fósforo em extratos de solos e plantas. Revista Ceres, Viçosa, v. 21, n. 113, p. 73-85, 1974.

BRANDÃO FILHO, J.U.T.; CALLEGARI, O. Cultivo de hortaliças de frutos em solo em ambiente protegido. Informe Agropecuário, Belo Horizonte, v. 20, n. 200/201, p. 64-68, 1999.

BREMNER, J.M. Total nitrogen In: BLACK, C.A., ed. Methods of soil analysis. Madison: American Society of Agronomy, 1965. part 2, p. 1149-1178.

CAMARGOS, M.I. Produção e qualidade de tomate longa vida em estufa, em função do espaçamento e do número de cachos por planta. Viçosa: UFV, 1998. 68 p. (Tese mestrado).

CASTELLANE, P.D. Constatação e interpretação fisiológica de diferenças de susceptibilidade de cultivares de tomateiro (Lycopersicon esculentum, Mill) à podridão apical. Viçosa: UFV, 1985. 183 p. (Tese doutorado).

CERDÁ, A.; MARTINEZ, V.; CARO, M.; FERNANDEZ, F.G. Effect of sulfur deficiency and excess on yield and sulfur accumulation in tomato plants. Journal of Plant Nutrition, v. 7, n. 2, p. 1529-1543, 1984. 
EL-JAOUAL, T.; COX, D.A. Manganese toxicity in plants. Journal of Plant Nutrition, v. 21, n. 2, p. 353-386, 1998.

FAYAD, J.A. Absorção de nutrientes, crescimento e produção do tomateiro cultivado em condições de campo e de estufa. Viçosa: UFV, 1998. 81 p. (Tese mestrado).

FURLANI, P.R.; BOLONHEZI, D.; SILVEIRA, L.C.P.; FAQUIN, V. Nutrição mineral de hortaliças, preparo e manejo de soluções nutritivas. Informe Agropecuário, Belo Horizonte, v. 20, n. 200/ 201, p. 90-98, 1999a.

FURLANI， P.R.; SILVEIRA， L.C.P.; BOLONHEZI, D.; FAQUIN, V. Cultivo hidropônico de plantas. Campinas: Instituto Agronômico, Campinas,1999b. 52p. (Boletim Técnico, n.180)

GERTSSON, U.E. Nutrient uptake by tomatoes grown in hydroponics. Acta Horticulturae, n. 401, p. 351-356, 1995.

HO, L.C.; ADAMS, P. Nutrient uptake and distribution in relation to crop quality. Hydroponic and transplant production. Acta Horticulturae, n. 396, p. 33-44, 1995.

INSTITUTO ADOLFO LUTZ. Normas analíticas do Instituto Adolfo Lutz. 3. ed. São Paulo,1985. v. $1,375 \mathrm{p}$.

KAFKAFI, V.; DAYAN, E.; AKIRI, B. Nitrate and phosphate uptake by tomato from nutrient solution in a commercial operation. In: INTERNATIONAL CONGRESS ON SOILLESS CULTURE, 6 ., 1984, Lunteren. Proceedings...Lunteren:. International Society of Soiless Culture, 1984. p. 291-298.

LEE, C. Mastering long shelf-life tomato growing. Pratical Hydroponics \& Greenhouses, n. 48, p. 85-89, 1999.
LOGENDRA, L.S.; JANES, H.W. Hydroponic tomato production. Growing media requirements. Acta Horticulturae, v. 2, n. 481, p. 483-486, 1999. MARTINEZ, H.E.P. Formulação de soluções nutritivas para cultivos hidropônicos comerciais. Jaboticabal: FUNEP, 1997. 31 p. (Boletim).

MARTINEZ, H.E.P. O uso do cultivo hidropônico de plantas em pesquisa. 2. ed. Viçosa: Imprensa Universitária, 1999. 47 p. (Cadernos Didáticos 1). MARTINEZ, H.E.P.; BRACCINI, M.C.L.; BRACCINI, A.L. Cultivo hidropônico do tomateiro (Lycopersicon esculentum, Mill). Revista UNIMAR, Marília, v. 19, n. 3, p. 721-740, 1997.

MORAES, C.A.G.; FURLANI, P.R. Cultivo de hortaliças de frutos em hidroponia em ambiente protegido. Informe Agropecuário, Belo Horizonte, v. 20, n. 200/201, p. 105-113, 1999.

MORGAN, L. Single truss tomato production in NFT systems. Pratical Hydroponics \& Greenhouses, n. 31, part 1, p. 42-47, 1996.

MORGAN, L. Single truss tomato production in NFT systems. Pratical Hydroponics \& Greenhouses, n. 32, part 2, p. 57-63, 1997a.

MORGAN, L. Fruit flavour and hydroponics. Pratical Hydroponics \& Greenhouses, n. 33, p. 21-31, 1997b.

MORGAN, L. Hydroponic fruit quality testing. Pratical Hydroponics \& Greenhouses, n. 34, p. 21-31, 1997c

MOURÃO, M.C.F. Interação boro x manganês em tomateiro (Lycopersicon esculentum, Mill). Viçosa: UFV, 1978. 34 p. (Tese mestrado).

NAKAMAE, I.J.; PASTRELLO, C.P. Tomate para a satisfação do consumidor. AGRIANUAL 99 Anuário da Agricultura Brasileira. São Paulo: FNP Consultoria \& Comércio, 1999. 489 p.
RÊGO, E.R. Herança da cor e caracterização de frutos de tomate mutante amarelo, normal, F1 e F2. Viçosa: UFV, 1997. 66 p. (Tese mestrado).

REUTER, D.J.; ROBINSON, J.B. Plant analysis - an interpretation manual. Melbourne, Sidney: Ikata Press, 1988. 218 p.

SAMPAIO, R.A. Produção, qualidade dos frutos e teores de nutrientes no solo e no peciolo do tomateiro, em função da fertirrigação potássica e da cobertura plástica do solo. Viçosa: UFV, 1996. 117 p. (Tese doutorado).

STEINER, A.A. The universal nutrient solution. In: INTERNATIONAL CONGRESS ON SOILLESS CULTURE, 6., 1984, Lunteren. Proceedings...Lunteren: International Society of Soiless Culture, 1984. p. 633-649.

TAKAHASHI, H.W. Relação Ca:Mg:Kno desenvolvimento, produção, composição mineral e distúrbios fisiológicos relacionados com o $\mathrm{Ca} \mathrm{em}$ tomateiro (Lycopersicon esculentum, Mill). Piracicaba: ESALQ, 1989. 167 p. (Tese doutorado).

WIEN, H.C. The physiology of vegetable crops. Cambridge: University Press, 1997. 662 p.

WINSOR, G.W. Some factors affecting the composition, flavour and firmness of tomatoes. Scientific Horticultural, n. 18, p. 27-35, 1966.

WOLF, B. Improvements in azomethine-H method for determination of boron. Communications in Soil Science and Plant Analysis, v. 5, n. 1, p. 39-44, 1974.

ZSCHEILE, F.P., PORTER, J. W. Analytical methods for carotenes of Lycopersicon species and strains. Analytical Chemistry, v. 19, n. 1, p. 47-51, 1947. 